\title{
Clinical evaluation led to a definitive diagnosis in $95 \%$ of patients presenting with dementia
}

\author{
Massoud F, Devi G, Moroney JT, et al. The role of routine laboratory studies and neuroimaging in the diagnosis of \\ dementia: a clinicopathological study.J Am Geriatr Soc 2000 Oct;48:1204-10.
}

QUESTION: In patients who present with dementia, what are the results of the dementia workup and how do these compare with the neuropathological diagnoses at autopsy?

\section{Design}

Cohort study with follow up to the death of the patient (mean follow up 4 y).

\section{Setting}

A tertiary care centre for patients with cognitive complaints in New York, USA.

\section{Patients}

61 patients (mean age 69 y, 59\% men) with cognitive complaints. Autopsy was available for the entire sample.

\section{Diagnostic strategy}

Patients had a clinical evaluation that included a structured neurological, functional, and psychiatric examination and a series of laboratory tests (complete blood count, serum electrolytes, liver and renal function tests, serum B12 folate, Venereal Disease Research Laboratory, and thyroid function tests). 60 patients had brain imaging (computerised axial tomography [CT] scan or magnetic resonance imaging [MRI] scans). The following were used: $D S M-I I I-R$ for dementia, NINCDS-ADRDA for Alzheimer's disease (AD), and NINCDS and the Association Internationale pour la Recherche et l'Enseignement en Neurosciences criteria for vascular dementia. The presence of concomitant $\mathrm{AD}$ with cerebrovascular disease on clinical evaluation or neuroimaging was classified as possible $\mathrm{AD}$. Mixed dementia referred to patients who had possible AD according to the NINCDS-ADRDA and concomitant cerebrovascular disease.

\section{Main outcome measure}

Pathological diagnosis (patients were followed to autopsy at which point they were assigned a pathological dementia diagnosis based on established pathological criteria).

\section{Main results}

41 patients $(67 \%)$ had a clinical diagnosis of probable $\mathrm{AD}$, $7(12 \%)$ had a diagnosis of possible AD, $10(16 \%)$ had another diagnosis (chromosome 17 associated familial dementia, frontal lobe dementia, Creutzfeldt-Jakob dementia, adult polyglucosan disease, Parkinson's disease associated dementia, and dementia of unknown aetio$\operatorname{logy})$, and $3(5 \%)$ did not meet criteria for dementia. Clinical and pathological diagnoses differed in 5 patients. 3 patients with a clinical diagnosis of $\mathrm{AD}$ had pathological diagnoses of pure Lewy body disease, subcortical gliosis, and multiple sclerosis; 2 patients with clinical diagnoses of dementia of unknown aetiology had $\mathrm{AD}$ on pathological examination.

\section{Conclusions}

In patients presenting with dementia, clinical evaluation that included a structured neurological, functional, and psychiatric examination; neuropsychological testing; and a series of laboratory tests provided a definitive diagnosis in 95\% (95\% CI 86\% to 99\%) of patients. Diagnoses were $67 \%$ probable Alzheimer's disease (AD), $12 \%$ possible $\mathrm{AD}$, and $16 \%$ another diagnosis.

\section{COMMENTARY}

The study by Massoud $e t a l$ adds to the literature related to (1) the sensitivity of recently developed, standardised clinical criteria for $\mathrm{AD}$ and vascular dementia compared with pathological diagnosis at autopsy; and (2) the diagnostic utility of neuroimaging (CT or MRI) for vascular dementia. The study also used a thorough laboratory workup, and in so doing detected abnormal results in $>15 \%$ of patients. None of these patients' cognitive functioning apparently improved with treatment of metabolic disorder, although methodological weaknesses severely limit conclusions related to reversible dementia based on these results.

Methodological strengths included rigour and comprehensiveness in the diagnostic assessments. Limitations included a lack of blinding of the outcome assessment, and a lack of information related to any dropouts or refusals. The study results are applicable to tertiary dementia clinics or other settings wherein the proportion of patients with dementia is high.

Practice recommendations based on this study are:

(1) The use of currently available structured diagnostic criteria for $\mathrm{AD}$ leads to good sensitivity and specificity compared with pathological diagnosis at autopsy, ${ }^{1}$ and as such this criterion set should be considered for routine clinical use.

(2) This study suggests that CT or MRI scanning is critical for the detection of vascular dementia (and mixed cases) and therefore should also be obtained, in the dementia workup, particularly if the management of the patient will need to be altered to include treatment of the cerebrovascular process (eg, stroke prophylaxis).

Robert van Reekum, MD, FRCPC Baycrest Centre for Geriatric Care Toronto, Ontario, Canada

1 McKhann G, Drachman D, Folstein M, et al. Clinical diagnosis of Alzheimer's disease: report of the NINCDS-ADRDA Work Group under the auspices of Department of Health and Human Services Task Force on Alzheimer's Disease. Neurology 1984;34:939-44.
Sources of funding: National Institutes of Health; Charles $S$ Robertson Memorial Gift for Alzheimer's Disease Research from the Banbury Fund; Blanchette Hooker Rockefeller Fund; McLaughlin Foundation.

For correspondence: Dr R Mayeux, Gertrude H Sergieusky Center, 630 West $168^{\text {th }}$ Street, New York, NY 10032, USA. Fax +1 2123052426. 